\section{CLINICAL LECTURE on}

\section{A CASE OF H E M I P L E G I A.*}

By J. HUGHLINGS JACKSON, M.D., F.R.C.P.,

Physician to the London Hospital, and to the Hospital for the Epileptic and Paralysed.

LECTURE I.

IN every case of hemiplegia, the important medical question is, How comes the patient to have that symptom? We must not dwell with exaggeration on the symptom itself; we must examine very carefully the patient who presents it. We ought never to lose sight of the fact that, in the majority of cases, nervous symptoms are results of old and slowly developed changes. Although a man may tell us that, on the day on which he became paralysed, "he never felt better in his life", we examine his heart, arteries, and indirectly his kidneys. In many cases, we discover evidences of long standing disease in some or all of these parts. Slow changes have been going on throughout him; and all that may have happened on the day the hemiplegia occurred was blocking up of an artery-only the small event that blood clotted on the roughened interior of a cerebral artery (thrombosis). But that artery - his arterial system, indeed-may have been years in preparation for such an occurrence. Do not take a nervous view of nervous diseases. An examination of the patient altogether, and a careful inquiry into his individual and family history, are the things of most importance. The patient's specially nervous symptoms, such, for example, as aphasia and hemiplegia, are, from a medical point of view, very often of less consequence than his general clinical state. Do not confound the two things, physiological interest and clinical importance. Aphasia is very interesting as a symptom; but the important thing is to obtain a clear knowledge of the bodily state of the person who presents that symptom and to find out how he comes to have it. Nay: not how he comes to have this or that symptom, but how it was that one of his cerebral arteries was blocked up or ruptured. These are the pathological processes almost always underlying aphasia. Indeed, most nervous symptoms are not directly of nervous origin. The most important and most common of them are of arterial origin : an artery bursts, and the escaped blood tears up a nervous centre; or an artery becomes blocked up, and a nervous centre softens. Nervous organs very often indeed suffer because their arteries are faulty. From a medical point of view, it is of the utmost importance to recognise this. From this standpoint, we see that things so different as hæmorrhage from the nose, hæmorrhage into the retinæe and into the corpus striatum, have often the very same pathological significance; they are all artcrial matters. These distinctions are not to be lost sight of in the consideration of heredity, as we shall see when we come to speak under the head of "Softening of the Brain".

I will not dwell on the constitutional state in the case of the subject of this lecture. Indeed, this patient's constitutional state was fair. $\mathrm{He}$ was young. Although he had aortic disease, he was not so much a diseased man as a locally damaged man. Why he had aortic disease, I could not ascertain. He had not had rheumatic fever, which, by the way, more often damages the mitral valves. Nor had he been subject to very hard work, involving strain-a common cause of, or factor in producing, aortic disease. Before leaving this part of the subject, I would remark that many of my patients who are hemiplecric, and who have valvular disease of the heart, have no serious heart-symptoms. This is worth stating, as it shows how accidental, so to speak, hemi plegia from the process of embolism may be. A man suffers hemiplegia, not because his circulation is defective, but because there are vegetations on the valves of his heart which can be detached. It is true that his valves may be so bad, that they do not fit; but there is compensation from hypertrophy of the heart's muscular walls, so that his circulation continues efficient. A patient begins to suffer from, to be incapacitated from, valvular disease, when the compensating hypertrophy fails. Do not forget this. A person will become hemiplegic who is, except for vegetations on his valves, in apparent good health There may occur embolism of a cerebral artery, and thence cerebral softening and local paralysis, without any premonitory symptoms, nervous or circulatory. At the most, the patient may have been a little

* On page 804 of last volume, will be found a report of the case which is the tex of this and subsequent Lectures. short of breath, almost certainly a little so, if he have mitral incompetence. Of course, embolism may happen at any stage of heart-disease. It may occur when a patient is slowly dying of the direct effects of heart-disease, but at any stage it is a sort of accident; and, I repeat, it often happens before there are any important heart-symptoms, and when the patient feels quite well, and is well, except for what is practically a latent mischief.

In all nervous cases, it is best to study first the Symptoms which Localise. In the case the text of this lecture, there are two localising symptoms, Hemiplegia and Affection of Speech. A patient does not understand how it is that, when he is paralysed, we pay attention first and most to his heart, arteries, and urine. It relieves his mind to enter fully into the symptoms which trouble him. We shall in this lecture discuss the hemiplegia first, and from this and the loss of speech we shall find out where the lesion is.

Hemiplegia is a symptom of loss of function of one lateral half of the motor tract. As this tract may be damaged at different levels, there are several anatomical varieties of hemiplegia. Thus there is hemiplegia from damage of one lateral half of the spinal cord, or of the pons Varolii, or of one of the crura cerebri; but I speak in this lecture of hemiplegia resulting from damage to one of the two highest divisions of the tract-the thalamus opticus and corpus striatum. This is the commonest kind of hemiplegia ; it is, indeed, the commonest kind of paralysis. It is the most important of all nervous symptoms. It is that paralysis which is found with affections of speech; after convulsive seizures (it is then called epileptic hemiplegia); often with cerebral tumour; very often as a consequence of cerebral hæmorrhage, or of local cerebral softening. It will occur at all ages; we find it in infancy (although it is not "infantile palsy"), in adults, and in very old people. I could give more reasons for saying that it is the most important of all nervous symptoms. Without a thorough knowledge of this symptom, the student is not fit to begin the scientific investigation of the more difficult nervous diseases, epilepsy, chorea, affections of speech, etc. We shall speak of it as the Common Form of Hemiplegia. It depends on destruction of the corpus striatum or of the optic thalamus. Observe the manner of statement. It is not scientifically exact to speak of it as a symptom of softening, or of cerebral hæmorrhage, or of tumour; for it is a symptom of destruction of the corpus striatum by any pathological process whatever. I should say "destruction of function", because, although in most cases there is actual breaking up of nerve-fibres and cells, yet in one clinical variety of hemiplegia there is only a temporary exhaustion of the nerve-fibres and cells of the corpus striatum. This is the epileptic hemiplegia of Dr. Todd. The following is my speculation* as to its mode of causation. There is in the convulsion a strong discharge of grey matter of convolutions through the corpus striatum to the muscles convulsed. If the discharge be excessive, the corpus striatum is left temporarily hors de combat; its nerve-fibres and cells are paralysed in the same way, I suppose, as a nerve can be experimentally paralysed by excessive artificial stimulation.

So, then, although it is true that cerebral hæmorrhage and softening are by far the commonest causes of hemiplegia, they are only some of the pathological processes which lead to destruction of the corpus striatum. Other processes are tumour and the temporary exhaustion just spoken of. The distinction is of more practical moment than appears at first glance. We shall not have clear views if we confound the functional change (here loss of function) with the pathological processes which lead to it. This confusion is more particularly to be regretted in the other class of functional changes ("instability"), as in chorea, epilepsy, etc.

The damage to the motor tract will vary in quantity. For example, there are clots in the corpus striatum of all sizes, from one of the bulk of a pea to one of the bulk of a small orange; there are roughly corresponding amounts of damage to the nervous centre. Hence the degree of hemiplegia resulting from clot, or from other kinds of destroying lesions, will vary exceedingly. It will vary from a slight and transient weakness of which the patient complains, but which examination almost fails to demonstrate, to complete and permanent hemiplegia. Here let me warn you not to conclude that a patient's hemiplegia necessarily depends on "functional" changes (in the sense that there is no structural alteration) because the paralysis was slight and transitory. Such slight and transitory palsy may depend on permanent although limited destruction by a very small clot, or softening from

* In this Jocknal, February 7 th, "After Effects of Epileptic Discharges", I have applied the principle of this speculation to the explanation of epileptic mania. The commonly received explanation of epileptic hemiplegia is, that the paralysis is a consequence of cerebral congestion, or actual extravasation in the brain, resulting from impeded respiration in the paroxysm. But I submit that this explanation is negatived by the fact, that absolute palsy of a part will folluw a limited epileptiform seizure in which there has been no trace of loss of consciousness, and nothing to indicate obstructed respiration. 
plugging of a very small artery. I shall afterwards prove to you that patients recover from hemiplegia notwithstanding that the paralysing restored.

I wish next to insist that the degree of paralysis in hemiplegia does not vary only with the quantity of damage. Introducing a more general term, I say that it varies with the Gravity of the lesion. There are two factors in the gravity of a nervous lesion; there is not only the quantity of nerve-tissue destroyed, there is also the suddenness with which the destruction is effected. For example, to take the two extremes, a large tumour and a large clot can differ, in that they destroy and squeeze different amounts of a nervous organ ; but they must differ, in that one destroys and squeezes very slowly, and the other very rapidly. So far as the production of nervous symptoms goes, clot and tumour, and also the several other pathological processes I have just been mentioning, differ chiefly as they differ in destroying slowly or suddenly. For the present, I shall ignore the pathological processes, and speak of "destroying lesions", or losses of function, of different degrees of gravity. One is justified in doing so, because there is nothing, or next to nothing, in hemiplegia, diagnostic of any pathological process. Hemiplegia from local softening of the corpus striatum does not differ from hemiplegia caused by clot in the corpus striatum; nor-and this I would impress upon you by repetition-does the hemiplegia from either of these changes differ from that sometimes found after a severe convulsion. It is very true that the mode of onset of hemiplegia is an exceedingly important element in the diagnosis of the pathological processes causing the symptom, as I shall tell you later on; but the hemiplegia itself is not.

We shall make three degrees of hemiplegia. The effects of a lesion of the third degree of gravity are, I believe, paralysis of both sides of the body. It is not then, strictly speaking, a degree of hemiplegia, but a degree of paralysis beyond hemiplegia. We shall, at any rate, consider the effects of lesions of three degrees of gravity in the two highest divisions of the motor tract. If it assist your imagination, you can think of clots of three different sizes, or of softenings from blocking of arteries of three different calibres. It follows from what has been said, that these degrees are arbitrary; but they are convenient. There are from lesions of different degrees of gravity, all degrees of symptoms, from slight one-sided numbness to universal powerlessness in deep coma (apoplexy). We begin with the first degree. What I call the first degree is that degree which you most often see; it is what we commonly call "hemiplegia" without any qualification. It is the degree our patient suffered from. We shall pay most attention to it.

\section{First Degree of Hemiplegia.}

As I have often told you, it is a good plan to examine a paralytic patient as it were geographically-from the first cranial nerve to the last, from his head to his feet. But to-day we shall pick out for remark the parts which we know to be affected in the first degree of hemiplegia. The parts affected are the face, tongue, arm, and leg, all of one side-the right side in our patient. Do not forget that all the paralysis there is is on the side of the body opposite the cerebral lesion. I mention this particularly, because in a justly esteemed work on physiology, it is said that the face is paralysed on the same side as the lesion, and therefore on the side of the body opposite that on which the arm and leg are paralysed. Now this sort of cross-paralysis only occurs in those lesions of one side of the pons Varolii, where the facial nerve is damaged in the pons before its decussation. In the form of hemiplegia we are considering, the lesion is anatomical miles above the decussation of the facial nerve, as well as above that of the limbs. The left will be the side of the brain diseased in our patient, as the right side of his body is paralysed.

Face.-Of the facial palsy in hemiplegia of the first degree, remem ber (I) that some is nearly always discoverable at least soon after the onset; (2) that it is slight, and often very transitory; (3) that the muscles affected are those supplied by the portio dura (facial) nerve (4) that the facial palsy is, as I have just insisted, on the same side as that of the limbs ; (5) lastly, observe that the palsy is not evenly distributed over the muscles supplied by the portio dura nerve. The last remark is important. If the trunk of the portio dura nerve be diseased, the palsy is evenly distributed over the face. Thus, if the disease be such that the nerve is destroyed, the face is considerably paralysed all over; but if part of the trunk only be destroyed, we do not find palsy of parts of one side of the face, but partial paralysis all over the face. (Of course, in wounds of the face involving twigs on the peripheral side of the main trunk, there may be paralysis of limited parts-as, for example, of the orbicularis palpebrarum.) Now, in the facial palsy of the common form of hemiplegia, the lesion is far above the trunk of the portio dura ; it is in a nerve-centre-the corpus striatum, for example -and this is very far above the decussation of the facial nerve in the pons. The palsy from disease of the corpus striatum is not evenly distributed ; the stress of it falls on the cheek, and although the orbicularis palpebrarum is affected, it suffers comparatively little. The patient can always shut his eye : it suffers so little that in many chronic cases we observe no difference in the closure of the two eyes, or only a trifling difference in the firmness of the closure when the patient tries to close his eyes tightly.

When our patient was asked to "show his teeth", the upper lip drew up to the left side; he could not close his right eye so firmly as the other, and the brow on the right side was less wrinkled on frowning. There was thus paralysis of muscles supplied by the portio dura nerve, or rather imperfect paralysis-paresis-the cheek suffering most. The facial paralysis in this case was more extensive and decided in range than is usual in hemiplegia, regard being had to the degree and continuance of paralysis of the arm and leg; I mean that the muscles (orbicularis palpebrarum and occipito frontalis) suffered more than is common. I believe this to be most often found in cases of hemiplegia from embolism; it is a fact of some slight empirical value in the diagnosis of softening by embolism from clot as a cause of hemiplegia.

Tonoue. - In hemiplegia the tongue suffers very little; there is slight deviation on protrusion, and the turning is to the side of the body paralysed. It is one of those things which educated malingerers overdo. It is well to contrast the paralysis of the tongue from lesion of the corpus striatum with the paralysis of this part from lesion of the lingual nervetrunk or its nucleus. In both cases, the tongue turns when protruded to the paralysed side. In hemiplegia, however, the tongue remains plump, and feels firm on both sides; but in cases of paralysis of one-half the tongue from disease of the lingual nerve-trunk, or of its nucleus, the tongue on the affected side is flabby, looks wasted, and after a time does waste. I have shown you several cases of this kind. Such a degree of palsy of the tongue never occurs with hemiplegia, so far as I have observed-that is, as part of the hemiplegia; it necessarily, I believe, indicates disease of the ninth nerve, or of its nucleus in the medulla oblongata, and on the same side as the palsy. The lingual palsy in hemiplegia is due to lesion very far above the medulla oblongata, and, as in the case of the facial palsy in hemiplegia, thelesion is far above the decussation, and on the opposite side of the brain. Paralysis of the tongue in hemiplegia differs as much from paralysis of the tongue due to lesion of the trunk of the ninth nerve, as does the facial palsy in hemiplegia from paralysis of the face due to lesion of the trunk of the portio dura nerve.

Remarks on Defective Articulation in Cases of Hemiplegia.-I must now speak of the influence of these trifling palsies on our patient's talking. He had loss of speech, or, as it is commonly called, aphasia. So far from causing loss of speech, they do not cause any considerable defect of articulation. I shall later on have to speak of affections of speech; just now, I speak of articulation only. You would not, perhaps, expect that such slight palsies of the face and tongue as we have just been describing would cause any great trouble in articulation. But I have good reasons for wishing to speak to you of the slight, the insignificant, effect on articulation of the paralysis of the face and tongue in the hemiplegia of adults. I will first, however, speak of cases of lingual and facial palsies, far severer than we ever see them in hemiplegia. Let us take, for instance, a case where the face is perfectly paralysed on one side, in disease of the trunk of the portio dura nerve, for instance, and let us suppose that there is also palsy and partial atrophy of the tongue on the same side. In such a case, the patient's articulation is very much less affected than you would, from theoretical reasoning, suppose. It is so little affected, that you have no difficulty in understanding all that he says. A fortiori, then, the trifling and often transitory palsies of the articulatory organs in hemiplegia can have little effect in impairing articulation; there is some transitory muffling soon after the attack, not from loss of power in the lips and tongue, but from sudden loss of power in these parts. Let me mention another fact about paralysis of the articulatory organs. A patient may articulate all the labials when he has absolute paralysis of all the superficial muscles of the face, including, of course, the lips. It is paralysis of the palate (on both sides) which interferes with the labials. It is very important indeed to bear such facts in mind. You would never, it is true, put down loss of speech or mistakes in words to such slight palsies of the face and tongue as occur in hemiplegia. But there is a kind of difficulty of articulation in some cases of right hemiplegia which, if you were very careless, you might put down to the slight paralysis of the face and tongue. This difficulty may be called ataxy of articulation, because, although the patient articulates very badly, he can move his lips, tongue, and palate apparently well in all other operations. See the seeming contradiction. Never forget that there occurs, from disease of one side of the brain, a great difficulty of articulation, in which we discover only the most trifling paralysis of parts of the articulatory organs ; so trifling is the discoverable paralysis, that it gives no explanation of the diffi- 
culty of articulation; the patient's talk is harder to be understood than that of a patient who has much obvious paralysis of the tongue, face, and palate on one side, and of one vocal cord from disease of the medulla oblongata. Be the explanation what it may, the facts are so.*

The difficulty of articulation in cases of disease of the medulla oblongata is a " thick" drunken-like talk; the articulation which is called ataxic is a sort of gabble. But there is an exceptional statement to be made; there is a thick, drunken-like talk with hemiplegia in cases of lesion of both sides of the brain. Let me explain. A patient has hemiplegia of the right side and gets well, talking well; he has next hemiplegia of the left side, and his talk is thick. This is because there is really a bilateral lesion; there is a recent one in the right motor tract to cause the left hemiplegia, and there is an old one in the left motor tract which caused the right hemiplegia. There is often, in these cases, some difficulty in swallowing, too. Later on, when speaking of Broadbent's hypothesis, I shall show you how it is that this thick speech occurs from a lesion of both motor tracts.

The ataxy of articulation without obvious, or with insignificant, paralysis of the articulatory muscles will remind you of Duchenne's ataxy of locomotion without obvious paralysis of the locomotor organs. They are both "disorclers of co-ordination". But to give this name to them is not to explain them, or it is an explanation which is simply verbal, and really explains nothing at all. $\dagger$

I shall have more to say of articulatory ataxy when I come to consider defects of speech.

Now we come to something puzzling. I told you that the lingual palsy, in cases of hemiplegia, was very little. But our hemiplegic patient could not put out his tongue when he tried, and thus it might seem that, in this case, the organ was perfectly paralysed. Nevertheless, there was really no such absolute paralysis as there seemed to be. The tongue moved quite well in such automatic or partly automatic processes as eating and swallowing. This state of things is frequently found in cases of loss of speech (aphasia). Now bear in mind that the inability is not to be explained away by saying that the patient does not understand what is asked of him. He opens his mouth, and, indeed, may move his tongue about in his mouth, drawing it back, for instance; he will even put his fingers in his mouth to get it out. After failure to protrude his tongue, by effort, he may put it out, to use a vulgarism, in a "promiscuous manner", as, for example, to catch a stray crumb. We may get it out now and then by a trick. Mr. Louis Mackenzie showed me that one of my aphasic patients, who could not put out her tonguc when we asked her, would put it out to lick her lips after taking a drink. Speechless patients have occasional difficulty in doing other things they try to do when told, as you will find to your trouble in some patients when, whilst using the ophthalmoscope, you tell them to look in certain directions.

It would have been a gigantic blunder to put down our patient's inability to talk to palsy of the tongue-to mistake loss of speech for los of power to move the tongue. A careless investigator might do this in a case of temporary loss of power to talk, with hemiplegia, as, for example, in a case of temporary loss of speech after a convulsion. A patient may lose speech wholly or partly for only an hour or two, or even for a shorter time, after some convulsive scizures (right-sicled). There is an "epileptic aphasia" as well as an "epileptic hemiplegia". If, as is likely, the patient could not then put out his tongue, you might, were you not warned, be deceived; you might suppose the affection of speech was owing to lingual paralysis. Let me show you further how you may discover that the tongue is really not paralysed in cases of loss of speech. Observe that although the rest of the palsy (face, arm, and leg) is on one sicle, the tongue, as in our patieut, "pretends" to be paralysed on both sides. Again, in nearly all cases. which we call loss of speech, there is power to utter some word or words, if not to use those words in speech ; occasionally it is a jargon only. Our patient could utter the words "yes" and "no" quite well, and he could not have done so had his tongue been as much paralysed on both sides as, so to speak, it pretended to be. But in a few cases of loss of speech, soon after the attack the patient is unable to utter any word, syllable, or jargon, and then this kind of test would not apply. I have had a patient under my care recently who has been speechless twelve months, who cannot utter any word or syllable; however, he makes a noise, which is possibly an * In some cases of ataxic articulation, there is disease of one side of the pons Varolii.

t I have long held that both in locomotor ataxy and in articulatory ataxy there is really a paralytic condition; there is, according to my hypothesis, weakening, not of muscles, but of some of the most special movements of the locomotor or articulatory series For example, 1 believe that in locomotor ataxy there is first weakening of that movementh which the peroneus longus is the muscle chienly concerned. The disorderly walk is explainable on the supposition that there is over estimate of the most special movement, and, consequently, over-development of associated movements - a state of things strictly comparable to that attending palsy of an ocular motor nerve ("erroneous projection" and "secondary deviation"). indistinct "aye" or "oh". We find, however, that the patient who cannot put out his tongue has the use of that organ, and of his lips and palate in all other processes than speech. The patient who cannot speak because his brain is diseased can swallow well, but the patient who cannot talk because his tongue is paralysed cannot swallow well. There is from swallowing a very obvious difference. We are constantly swallowing our saliva ; the patient whose tongue is paralysed cannot do this, hence he carries his pocket-handkerchief in his hand, because his saliva dribbles. ${ }^{*}$ There is still another test-viz., that the patient who is unable to talk, simply because he cannot move the muscles of articulation from actual palsy of them, can write, i.e., can express himself in writing. In short, such a man can speak, for we must speak internally before we write. But the patient who is properly called speechless has, I consider, neither external nor internal speech ; obviously, he cannot talk aloud; he never does; but he cannot speak anyhow, not even to himself, or he would write what he speaks to himself. The speechless man may sign his name ; he may also copy writing. There is no reason why he should not, as this does not require speech. $\mathrm{He}$ cannot read what he has copied. Our patient had never learned to write, and thus this test was inapplicable in his case.

Let us put some of these diagnostic points together in another way. If you were told of a patient who could not "talk", but who could swallow well, and could not write, you would say, "This so-called inability to talk is really loss of speech from disease of the brain. If you were told that a patient who could not speak, but could write well, and yet could not swallow well, you would say that strictly his defect was not loss of speech, but loss of pozver to move his articulatory organs. You may diagnose such cases from a distance; the patient carries his pocket-handkerchief in one hand, and a small slate hangs from his button-hole. What are we to say of the tongue, which the patient cannot put out when he tries hard to do so? He has lost the most voluntary or most special movements of it. This may appear to you simply repeating in technical phraseology that he cannot put it out when he tries. But at all events he has lost only some of the movements of it, its muscles serving well in other movements : he has lost the most special movements of it. We shall see that it illustrates a principle.

There was, doubtless, in our patient slight actual paralysis of the tongue, such as in cases of hemiplegia, without loss of speech, causes deviation on protrusion. Plainly, this could not be demonstrated, since he could not put his tongue out. The slight palsy, if it existed-as no doubt it did-was drowned, so to speak, in the larger loss of voluntary power over the whole organ. When, as usually happens in cases of loss of speech, a patient becomes able to put out his tongue, we may see a slight deviation of it.

So far, then, we have found that there is paresis of the face and tongue on their right side. These palsies, I repeat, are usually slight, and frequently transitory.

[To be continued.]

\section{CLINICAL MEMORANDA.}

\section{CASE OF OCCLUSION OF GALL-BLADDER.}

ON June 20th, I was summoned suddenly to see Mary R., aged 29, who had bcen confined three weeks. On my arrival, I found her to be lead. She had been under my care some weeks previously to her confinement, suffering with pleurisy and anasarca, which yielded to ordinary treatment. On performing the post mortem examination thirty-six hours after death, we came to the conclusion that the immediate cause of death was serous apoplexy of a most marked character. On examination of the abdominal viscera, the liver was found to be enormously enlarged, to the weight of nearly ten pounds; the structure was tolerably healthy. The gall-bladder was filled with calculi, to the number of two hundred and thirty-seven; the aggregate weight being 420 grains. The calculi were an almost exact representation, in size, appearance, and colour, of grains of Indian corn, rounded on their outer surfaces next the gall-bladder, and flattened internally by their compression and packing. There was no form of jaundice. The ductus communis choledochus was patent; and the circulation of bile in the gall-bladder could only have been carried on in the interstices of the outer surfaces of the calculi. During my knowledge of the case, there was no history of any dyspepsia, constipation, or hemorrhoids.

G. E. Corrie JACkson, L.R.C.P.E., M.R.C.S.Eng., Medical Officer, Westminster Union.

* I must say, however, that this dogma does not apply to children who have no speech, or whose speech is defective, from damage to the brain in early infancy. There is often pscudo-salivation in these cases. 